\title{
A whole-brain imaging-based systems approach to understand origin of addiction in binge-like drinking model
}

\section{Marzena Stefaniuk, ${ }^{1}$ Monika Pawłowska, ${ }^{1,2}$ Klaudia Nowicka, ${ }^{1}$ Marcin Barański, ${ }^{1}$ Zbigniew Zielinski, ${ }^{1}$ Lukasz Bijoch,,${ }^{1,3}$ Diana Legutko, ${ }^{1}$ Piotr Majka, ${ }^{4}$ Sylwia Bednarek, ${ }^{4}$ Natalia Jermakow, ${ }^{4}$ Daniel Wójcik, ${ }^{4}$ Leszek Kaczmarek ${ }^{1,}$ *}

${ }^{1}$ Laboratory of Neurobiology, Nencki Institute, BRAINCITY, Warsaw, Poland; ${ }^{2}$ Institute of Experimental Physics, Section of Optics, Warsaw University, Warsaw, Poland; ${ }^{3}$ Laboratory of Neuronal Plasticity, Nencki Institute, BRAINCITY, Warsaw, Poland; ${ }^{4}$ Laboratory of Neuroinformatics, Nencki Institute, Warsaw, Poland

*Corresponding author: Leszek Kaczmarek, Nencki Institute, BRAINCITY, Warsaw, 02-093, Poland. Email: 1.kaczmarek@nencki.edu.pl Phone: +48 225892240

Running title: Brain reorganization in binge-like drinking mice

Keywords: alcohol/addiction/binge drinking/light-sheet microscopy/optical tissue clearing 


\begin{abstract}
Many fundamental questions on addiction development are still unanswered. These questions are frequently difficult to address by examining a single brain structure, but can best be addressed at the systems level. Neurons create functional networks that change over time, since brain regions may work together differently in different contexts. We offer a framework for describing the nature behind alcohol binge drinking and the transition to addiction. The present study investigated whole-brain c-Fos expression following reexposure to alcohol in a model of binge-like drinking in mice in IntelliCage. We developed a dedicated image computational workflow to identify c-Fos-positive cells in three-dimensional images obtained after optical tissue clearing and whole-brain imaging in the light-sheet microscope. We analyzed functional networks and brain modularity following reexposure to alcohol. c-Fos levels in brains from animals that were reexposed to alcohol were clearly different from binge drinking animals. Structures involved in reward processing, decision making and characteristic for addictive behaviors stood out particularly. In alcohol reexposed animals differently active structures either gained or lost correlation when compared to the control group.
\end{abstract}




\section{Introduction}

Binge drinking is a type of alcohol use disorder (AUD) that involves consuming an excessive amount of alcohol in a short time (2004). Binge drinking episodes may turn into heavy drinking and increase the likelihood of developing addiction (Dawson et al, 2005). In alcohol addiction, cues that are associated with alcohol trigger craving and the relapse (George \& Koob, 2010; Namba et al, 2018). Alcohol induces multiple alterations in the brain that are thought to contribute to addiction, but an understanding of the neuronal ensembles responsible for excessive alcohol drinking is still preliminary (George \& Hope, 2017).

Whole-brain mapping techniques produce large datasets of anatomical and functional connectivity patterns (Sporns \& Betzel, 2016). The state of functional brain networks may influence the ways in which the brain manages information and changes in result of experience (Rubinov \& Sporns, 2010). Strong evidence shows that brain networks have a modular organization [for review, see (Bullmore \& Sporns, 2009)]. Such modules are thought to represent groups of brain regions that are collectively involved in specific functional domains (e.g., supporting cognition) (Sporns \& Betzel, 2016). Brain network properties, such as modularity, provide an important dimension for understanding the brain in health and disease (Arnemann et al, 2015; Liu et al, 2019; Meunier et al, 2009; Zhang \& Volkow, 2019).

Whole-brain imaging in human subjects has advanced our understanding of the effects of alcohol on the brain, but its use has been limited by ethical considerations, the heterogeneity of alcoholic populations, and the low spatial resolution of imaging methods (Zahr \& Pfefferbaum, 2017). In contrast, animal models enable the control of multiple genetic, environmental, and alcohol consumption factors (Fuchs et al, 2019; Spanagel, 2017). By mimicking neuropsychiatric criteria, such models help mimic patterns observed in humans (Sanchis-Segura \& Spanagel, 2006; Skora et al, 2020). Furthermore, spatial resolution of the imaging approaches that are available for studying animals' brains are far superior over 
those available in humans (Martin, 2014). Identifying neurons, their pathways and networks that modulate binge drinking may provide insights into what happens when a subject is occasionally exposed to alcohol. Thus, this knowledge may eventually lead to developing therapies against addiction.

The present study sought to provide a global analysis of the pattern of binge-like drinking-related c-Fos expression in the brain in mice. c-Fos is a widely used marker of neuronal activation that mediates the long-term potentiation of excitatory synapses (Knapska et al, 2007; Sun et al, 2020). We adapted the drinking-in-the-dark (DID) binge-drinking paradigm (Rhodes et al, 2005; Thiele \& Navarro, 2014) to the IntelliCage training system. This behavioral setup allows the long-term monitoring of animals that live in social groups with minimal contact with experimenters (Beroun et al, 2018; Kiryk et al, 2020; Koskela et al, 2018; Radwanska \& Kaczmarek, 2012; Smutek et al, 2014; Stefaniuk et al, 2017). Training consisted of initial acquaintance with alcohol that was presented concomitantly with a visual cue, followed by a withdrawal period and next by a session that exposed the mice to either the cue alone or the cue with alcohol access, as during the initial training. To assess cFos expression that was triggered by reexposure, the brain tissue was collected $2 \mathrm{~h}$ after alcohol administration. Importantly, c-Fos expression typically follows a very specific time course, in which protein is detectable in neuronal nuclei within $\sim 1-2 \mathrm{~h}$ after a stimulation (Knapska et al., 2007). To reveal brain-wide neuronal activation patterns, whole brain hemispheres were optically cleared and immunostained for c-Fos. Clearing removes lipids to render the tissue transparent (Chung et al, 2013; Renier et al, 2014; Susaki et al, 2015). Cleared hemisphere were imaged in toto using a light-sheet microscope (Pawlowska et al, 2019). The signal intensity of c-Fos-positive nuclei was transformed into the signal density that was specific to each brain structure. These were next used to examine whether reexposure 
bioRxiv preprint doi: https://doi.org/10.1101/2021.02.17.431586; this version posted March 1, 2021. The copyright holder for this preprint (which was not certified by peer review) is the author/funder, who has granted bioRxiv a license to display the preprint in perpetuity. It is made available under aCC-BY-NC-ND 4.0 International license.

to alcohol or the alcohol-associated cue following long-term withdrawal resulted in alterations of c-Fos expression, functional networks, and brain modularity. 


\section{Results}

Adaptation of the drinking-in-the-dark model to the IntelliCage training system

We employed an animal model of binge-like alcohol drinking, referred to as DID (Thiele \& Navarro, 2014) that was adapted for the IntelliCage (Fig. 1A). This behavioral training system allows the tracking of corner visits, nosepokes that permitted access to and licks from the drinking bottles located in the corners (Fig. 1B). Only one mouse could be present in a corner at a time. Previously, the usefulness of the IntelliCages for modeling AUD has been demonstrated (Kiryk et al., 2020; Radwanska \& Kaczmarek, 2012; Stefaniuk et al., 2017).

In the original DID design that was reviewed by Thiele and Navarro (Thiele \& Navarro, 2014), mice were housed individually. Three hours into the dark phase of the diurnal cycle, instead of water, they were given access to $20 \%$ alcohol for $2 \mathrm{~h}$. The procedure lasted for 4 days. At the end of each drinking session, blood samples were collected to measure blood alcohol levels (Cox et al, 2013).

In the present experimental design, we followed the same timeframe of drinking sessions ( $2 \mathrm{~h}$ session starting $3 \mathrm{~h}$ into the dark phase of the light/dark cycle), but the mice were kept in groups and did not undergo the sessions individually. Five seconds of access to alcohol was provided after each nosepoke, which was always associated with a visual cue (Fig. 1B). We extended the DID sessions to 3 weeks (with no access to alcohol during weekends as per original protocol). For the next 2 weeks, we limited access to alcohol to one corner with concurrent access to water in another corner. It was followed by a 9-day withdrawal with no access to alcohol. Finally, the mice were exposed to either 2-h access to alcohol that was paired with a light cue or the cue only in the corner that was previously associated with alcohol (Fig. 1A). Importantly, we did not attempt to measure blood alcohol 
levels to exclude possible confounding effects of manipulation, such as stressing the animals by separating them from the group.

Mice exhibit an increase in craving for alcohol after drinking sessions

During pre-binge drinking period, the mice explored the cage and had free access to drinking water in two corners. They learned to make a nosepoke to access the drinking bottles. Next, the mice were given access to $20 \%$ alcohol, combined with a visual cue, that was provided in the corners. During each drinking session, we measured the number of alcohol licks made by each mouse. This measure was used as a proxy of alcohol consumption. Throughout the experiment, the animals consumed alcohol voluntarily during daily 2-h sessions, reaching an average consumption of $3.82 \pm 0.88 \mathrm{mg} / \mathrm{kg}$ of body weight per $2 \mathrm{~h}$ per day, when alcohol was available in two corners, and $3.57 \pm 1.23 \mathrm{mg} / \mathrm{kg}$ of body weight when alcohol was limited to one corner. With our DID protocol, the animals developed craving for alcohol. This was manifested by a marked increase in the number of nosepokes in the alcohol corners during the 2-h period immediately following the drinking session (Fig. 1C, D). Throughout the drinking sessions, the mice made an average of $16 \pm 3$ nosepokes in the corners. During the $2 \mathrm{~h}$ immediately before the drinking sessions, they made on average of 10 \pm 2 nosepokes in the corners. Notably, $2 \mathrm{~h}$ after the drinking session, they made an average of $33 \pm 7$ nosepokes in the corners, indicating high levels of craving for alcohol. The number of nosepokes between these three time periods was significantly different (Fig. 1C). We observed a similar result when we analyzed the average number of nosepokes per session (Fig. 1D). During weekends, when alcohol was not provided, these seeking patterns were also maintained. These results indicate that the animals actively sought alcohol, predominantly immediately after binge-like drinking sessions. 


\section{Mice prefer alcohol over water during reexposure to alcohol or the alcohol-related cue}

After the drinking period, the animals were deprived of access to alcohol for 9 days, followed by reexposure. To verify that behavior during reexposure was unrelated to overall activity, we performed an analysis of the correlation between the number of nosepokes during this period and the number of all nosepokes in all corners during the entire experiment and found no correlation (Supplementary Fig. S1).

Under reexposure conditions, we measured the number of nosepokes in the alcohol corner and water corner and compared them to the corresponding timeframe during the withdrawal session that considered activity during the last day of withdrawal. In the CR group, a cue (light) appeared following a nosepoke, but no alcohol reinforcement was provided. In the AR group, each nosepoke opened the door to grant access to alcohol for $5 \mathrm{~s}$ simultaneously with the visual cue. Animals in both groups preferred the alcohol corner over the water corner (average of $59 \pm 36$ and $12 \pm 4$ nosepokes in the alcohol corner and water corner, respectively, in the CR group; average of $41 \pm 15$ and $6 \pm 5$ nosepokes in the alcohol corner and water corner, respectively, in the AR group, Fig. 1E). These results indicate that mice that were exposed to alcohol and the alcohol-associated cue in the binge-like drinking model exhibited a preference for the alcohol corner, suggesting that they associated the alcohol corner and the alcohol-related cue with alcohol reward.

\section{Whole-hemisphere c-Fos expression profiling in binge-like drinking mice}

To examine whether reexposure to alcohol or the alcohol-related cue following withdrawal after prolonged binge-like drinking resulted in changes in c-Fos expression, we performed optical tissue clearing and c-Fos immunohistochemistry (Renier et al., 2014). To prepare a map of brain areas that responded to either alcohol plus the cue or the cue alone, we 
aligned the brain structure distribution of the c-Fos signal with the Allen Brain Atlas (2017).

The overall result was a three-dimensional map of c-Fos-positive puncta with specific anatomical annotations (see Supplementary Movie S1). This map was converted to a c-Fos signal density map that was specific for each animal. A hierarchical list of identified structures was used for comparisons between groups, correlation analysis, structural modularity analysis and network analysis.

Several brain structures differentially expressed c-Fos in response to specific experimental conditions (Supplementary Dataset S1). These included structures that are implicated in motor function, visual and olfactory areas, the thalamus, and other. The most prominent differences were found between the AR group and BD and CTRL groups. The cFos signal density was much higher in the emotional response-related infralimbic area (ILA), decision-making-related anterior cingulate (ACA), motor function-related cuneiform nucleus (CUN), motivation process-related pericommissural nucleus (PRC), and memory-related postrhinal area (VISpor) in the AR group compared with the CTRL and BD animals. For statistical details for other structures, see Supplementary Dataset S1 and Fig. S2-S5.

\section{Alcohol reexposure but not binge-like drinking induces activation of the amygdala}

c-Fos expression in the amygdala in rats was previously found to be markedly elevated following reexposure to alcohol-related cues (Radwanska et al, 2008). In the present study, we noted a clear difference in c-Fos expression in the AR group, particularly in amygdala nuclei and their afferent and efferent structures. The central nucleus of the amygdala (CEA), basolateral amygdala anterior part (BLAa) exhibited a higher c-Fos signal density in the AR group compared with the BD group (Fig. 2). Another structure that was prominently activated by alcohol reexposure was the bed nucleus of stria terminalis (BST), a major output pathway of the amygdala, and the ventral tegmental area (VTA), an amygdala input. One of the richest 
areas of c-Fos-positive nuclei in the AR group was the anterior cingulate area (ACA), a structure that provides a strong input to the BLA. For detailed comparisons between the BD and AR groups, see Fig. 3. For comparisons between the other groups, see the Supplemental Information. These results indicate that during reexposure to alcohol after withdrawal, the whole amygdala complex and its input and output structures are strongly activated, reflected by c-Fos expression, compared with BD animals.

\section{Reexposure to alcohol alters functional neuronal co-activation}

To examine whether reexposure to alcohol or the alcohol-related cue resulted in functional network alterations, we used data on c-Fos profiling and performed structural coactivation analysis by calculating Pearson correlation matrices for each group separately. The ordering of structures in these matrices followed the anatomical groups from the Allen Brain Atlas (2017). The following gross divisions of the brain were applied: cortical plate, cortical subplate, striatum, pallidum, thalamus, hypothalamus, midbrain, and hindbrain. We observed similar patterns of coactivation in the CTRL and CR groups (Fig. 4A, C). Binge-like drinking mice exhibited a lower level of cross-correlation between various brain regions compared with the CTRL group (Fig. 4B). The AR group exhibited a prominent anticorrelation between more brain regions compared with all of the other groups, with the exception of hypothalamus nuclei, which had a strong interregional positive correlation (Fig. 4D). Reexposure to alcohol was shown to activate the amygdala; thus, we analyzed functional connectivity of amygdala nuclei with other brain regions and found that the amygdala became either poorly or negatively correlated in the AR group (Fig. 4E). For details see the Supplement. These results indicate that the activity of brain regions in response to alcohol was less correlated globally compared with alcohol drinking, binge drinking, or exposure only to 
the alcohol-related cue. Reexposure to alcohol induced the most prominent and distinctive brain-wide changes.

\section{Reexposure to alcohol increases structural modularity of the brain}

Brain networks are information processing systems that share a modular community structure (Meunier et al, 2010). A network is modular when sets of structures form densely interconnected groups with sparser connectivity between groups (Newman, 2006). Brain modularity alterations have been described in numerous brain disorders (Arnemann et al., 2015; Liu et al., 2019) and also upon withdrawal from alcohol consumption (Kimbrough et al, 2020). To examine whether reexposure to alcohol or the alcohol-related cue following withdrawal after prolonged binge-like alcohol drinking resulted in changes in brain modularity, we performed hierarchical organization analysis (Fig. 5). We calculated the Euclidean distance between rows of the correlation matrix and performed hierarchical clustering (Fig. 5). Heatmaps were generated with dendrograms in which the height at which any two structures were first connected corresponded to their degree of similarity. To compare dendrograms of the different experimental groups, we plotted the number of clusters based on relative tree heights. The hierarchical cluster dendrograms were next cut at half the height of each given tree to split the dendrogram into specific modules. In control animals, the analysis revealed that the brain was organized into three modules (Fig. 5A). In mice that were exposed to alcohol, the brain was organized into seven modules in the BD group (Fig. 5B), eight modules in the CR group (Fig. 5C), and 12 modules in the AR group (Fig. 5D). This indicates that binge-like drinking itself increased brain modularity. Reexposure to alcohol following withdrawal increased hierarchical modularity of the brain even further (Fig. 5E).

We also found that the amygdala and its input and output structures that were shown to be active with reexposure to alcohol shared one module in CTRL, shared two modules in 
BD animals, and fell into different modules in AR animals, thus indicating a major network rearrangement (Supplementary Dataset 2 and Fig. S11-14).

Finally, we investigated whether we could distinguish any specific functional module with components that are involved in substance use disorder or reward circuitry in the AR group (Koob \& Volkow, 2016). We identified two modules that included five structures that are implicated in addiction (i.e., ventral tegmental nucleus [VTN], temporal association area [TEa], BST, superior colliculus [SCiw], and nucleus accumbens [ACB]). Interestingly, when comparing c-Fos density profiles in the AR group, the TEa, BST, SCiw, and ACB had a higher level of c-Fos accumulation compared with binge-like drinkers (see Fig. 3). We also identified another potentially relevant module whose structures are implicated in rewardseeking behavior. This module included the substantia innominata (SI), retrohippocampal region (RHP), interfascicular raphe nucleus (IF), and BLAa. The BLAa is one of the major components in this module, together with the IF and subiculum (part of the RHP). Other amygdala components shared one module (medial amygdala [MEA], subparafascicular nucleus [SPF], vestibular nuclei [VNC], CEA, postpiriform transition area [TR], parastrial nucleus [PS], red nucleus [RN], intercalated nuclei [IA], pedunculopontine nucleus [PPN], supratrigeminal nucleus [SUT], subfornical organ [SFO], ectorhinal [ECT], and visceral area [VISC]).

These results indicate that alcohol reexposure implicates disconnection of functional networks of the brain toward the formation of fine circuitries that are involved in the development of addiction and processing of emotional information. Importantly, no such rearrangements were observed in the control group or binge-like drinking animals after the last drinking session.

Network fragmentation in alcohol reexposed brain 
To assess whether modular organization of the cue and alcohol reexposed brain network was related to neurobiological organization of the structures relevant to addiction (Koob \& Volkow, 2016), we employed a functional network analysis, as a collection of nodes (structures) and edges (links) between pairs of nodes (Rubinov \& Sporns, 2010). Individual nodes and edges differ in their impact on the overall functioning of the network. Some nodes are more essential or more influential than others. Some edges carry more traffic. Important nodes are often more highly and densely connected to the rest of the network facilitating global integrative process (Sporns, 2010). First, to identify interaction between all structures we calculated within-module degree z-score (WMD) and the participation coefficient (PC). The role of each node was defined by both of those values (Guimera \& Nunes Amaral, 2005) (Fig. S15), where WMD sets the importance of structure within its module and PC the importance of outside module connections. All nodes were classified into four distinct groups: provincial hubs (likely to play an important part in the facilitation of modular segregation), connector hubs (highly relevant outside and inside module), non-hub connectors (likely to facilitate global intermodular integration) and peripherals (with no role in within or outside connectivity) (for details see Supplementary Methods). We noticed that in reexposure groups some structure gain functionality facilitating intermodular integration (Fig. S15).

Next, we selected brain structures relevant to addiction development: ACB, AI, BLA (divided into BLAa, BLAp, BLAv), BST, CEA, GPe, GPi, HIP, IA, SNc, VTA (Koob \& Volkow, 2016) and performed functional connectivity analysis (Fig. 6). In animals living in homecages with no access to alcohol, spontaneous "resting-state" functional connectivity state revealed most structures were equally connected with similar strength (Fig. 6A). After twohour drinking session a pattern of large functional connectivity network changed with Globus Pallidus segments being slightly detached (Fig. 6B). While exposure to alcohol matched cue following withdrawal did not significantly changed the network (Fig. 6C), alcohol reexposure 
following withdrawal caused a major disconnection of functional network of the brain (Fig. 6D). Reexposure to alcohol implicated the disruption of functional interactions between structures creating specific patterns where BST and VTA shared the same pattern and it is different from GPe, BLAp, BLAv, HIP, IA, SNc and CEA pattern (Fig. 6D). We found that the extended amygdala structure CEA and BST and cortico-striatal loops still interacted with the mesolimbic dopamine region, however, the relationship is much weaker with almost no interactions with structures within the region. Interestingly $\mathrm{ACB}$ did not interact through positive connectivity with any other addiction relevant structure (Fig. 6D). 


\section{Discussion}

In this study, we characterized global brain c-Fos activity in a mouse model of bingelike alcohol drinking. First, we evaluated binge-like drinking behavior in IntelliCage. Next, we identified brain regions that exhibited alterations of c-Fos signal density following reexposure to alcohol, using whole-brain imaging technique on optically cleared tissue. We observed different brain structure correlation patterns in naive animals and alcohol- or cuereexposed animals. Lastly, we observed an increase in brain modularity and decrease in functional connectivity in animals that were reexposed to alcohol, compared with all of the other groups.

To characterize the binge-like drinking phenotype in mice, we adapted the DID model (Rhodes et al., 2005; Thiele \& Navarro, 2014) to the IntelliCage system. This behavioral training and testing system was previously used to model alcohol addiction in mice (Koskela et al., 2018; Radwanska \& Kaczmarek, 2012; Smutek et al., 2014; Stefaniuk et al., 2017). To date, however, no study has used the IntelliCage to study binge-like alcohol drinking.

The DID assay provides a $20 \%$ alcohol solution to mice for a limited time each day. In the original protocol, exposure to alcohol was limited to $2 \mathrm{~h}, 3 \mathrm{~h}$ into the dark phase of the diurnal cycle, for 4 days, accompanied by daily measurements of blood alcohol levels. In the current experiment, we extended the procedure and omitted blood alcohol level measurements to reduce stress in the animals. The use of IntelliCage allowed tracking individual mice movement and calculating alcohol consumption with high accuracy, that reached levels consistent with experiments which used standard DID method (Rhodes et al, 2007; Wilcox et al, 2014). Even with concurrent access to water in the last DID session, the animals still reached similar levels of alcohol consumption. A similar observation was made in a previous study (Giardino \& Ryabinin, 2013). 
Using the IntelliCage DID training paradigm, we found an increase in alcohol craving immediately after binge drinking session. The persistence of seeking alcohol has been previously used in other models of AUD as a measure of craving (Giuliano et al, 2018; Radwanska \& Kaczmarek, 2012; Stefaniuk et al., 2017); for review, see (Luscher et al, 2020). Furthermore, the mice exhibited an increase in preference for either alcohol or the cue alone following reexposure after withdrawal. Similarly, previous studies showed that a history of alcohol drinking during DID increased alcohol preference (Cox et al., 2013; Wilcox et al., 2014). Considering the complexity of AUD and the number of protocols that are used to model it based on various aspects of drinking patterns, our approach may facilitate further studies of the transition to addiction in automated controlled systems, such as the IntelliCage.

To create a map of brain structures that responded to either alcohol or the cue following withdrawal, we used c-Fos expression as a proxy of neuronal activation. c-Fos is a transcriptional factor that is activated upon neuronal stimulation (Knapska et al., 2007; Sun et al., 2020; Yap et al, 2020). Transcriptional modifications are postulated to be crucial for the development of addictive states (Nestler, 2001). Alterations of gene expression would lead to lasting modifications of neuronal activity and ultimately changes in neural circuits and stable changes in behavior. c-Fos has been reported to be activated after reexposure to alcohol (Smith et al, 2019) and alcohol-related cues (Radwanska et al., 2008). The cue- and contextinduced reinstatement of seeking alcohol induces the expression of c-Fos in discrete populations of neurons in various brain regions (Hamlin et al, 2009; Hamlin et al, 2007; Marinelli et al, 2007; Millan et al, 2010). Nonetheless, an overall picture of c-Fos expression is still lacking. In the present study, to trigger c-Fos expression, the mice were exposed to either alcohol or the cue after a 9-day withdrawal. Our analysis revealed a complete map of structures that were characterized by different activity profiles, reflected by the density of cFos expression. A similar approach was used after antipsychotic treatment with haloperidol 
(Renier et al, 2016), the regulation of energy expenditure (Schneeberger et al, 2019), alcohol abstinence (Kimbrough et al., 2020) and opioid relapse (Keyes et al, 2020).

The present study advances the c-Fos mapping technique by introducing c-Fos signal density analysis with more accurate anatomical details that cover many structures and layers. For instance, we identified various densities of c-Fos signals in different layers and parts of the ACA in animals that were reexposed to alcohol. Noteworthy, ACA in humans has been reported to contribute to motivational valence assignment (Mesulam, 1990) and reward assessment (Knutson et al, 2000). Other structures that are markedly activated in animals that are reexposed to alcohol include nuclei of the amygdala that have been previously implicated in reinforcing drug craving (Ryabinin et al, 1997) and addiction in studies in human patients (Goldstein \& Volkow, 2002; Koob \& Volkow, 2016) and animal models (Augier et al, 2018). Markedly altered were also input and output structures of amygdala nuclei implicated in drug seeking (Belin et al, 2013). The global brain c-Fos profiling approach provides information about particular structures and their neuronal ensembles. Such profile of neuronal activation may shed light on addiction with regard to notable brain regions and specific patterns of sparsely distributed neurons (i.e., neuronal ensembles) that are selectively activated by specific cues, contexts, and rewards during alcohol-related behavior (George \& Hope, 2017).

Further analysis of c-Fos density correlation maps enabled comparisons between groups of structures. All such groups exhibited specific correlation patterns, with high positive correlations in control binge-like drinking animals and cue-reexposed animals. Following reexposure to alcohol, groups of structures were highly negatively correlated. This indicates a significant reconstruction of the global brain activity pattern. A recent study showed that such rebuilding is also observed during abstinence, although in the opposite direction (i.e., positive co-activation) (Kimbrough et al., 2020). In the present study, however, 
the trigger of c-Fos expression was different and had a well-defined onset (i.e., reexposure to alcohol).

Using hierarchical clustering, we found an increase in brain modularity in the AR group (12 functional networks/modules) compared with the BD, CR, and CTRL groups showing seven, eight and three modules respectively. Changes in brain modularity have also been observed during abstinence (Kimbrough et al., 2020), with a decrease in brain modularity. We hypothesize that reexposure to alcohol can be a strong behavioral stimulus that is sufficient to trigger major changes in functional brain architecture. Kimbrough et al. (Kimbrough et al., 2020) reported that a decrease in modularity might be partially responsible for cognitive dysfunction seen in humans and animal models of alcohol dependence, but this does not appear to be the case in binge-like drinking models. Human magnetic resonance imaging studies indicate that network organization into higher modularity and thus connectivity within modules and sparse connections between modules may reflect more effective signaling across brain regions (Stevens et al, 2012). We suggest that the increase in brain modularity that was observed under the present experimental conditions might be transient and limited selectively to reexposure to alcohol and not to alcohol consumption itself, in which BD animals had lower modularity than the AR group.

Finally, we aimed at connecting practically all structures into interacting network defining function of components within the network (Sporns, 2010). We described the effective strength of functional interaction and contributions of individual nodes determined by their interaction within a local and global neighborhood (Guimera \& Nunes Amaral, 2005). We did not identify any major hub structures within functional modules in reexposure groups. We next assessed the way in which the modular organization of the reexposure network was related to the neurobiological organization of the addiction-relevant brain structures (Koob \& Volkow, 2016). Control animals exhibited spontaneous "resting-state" functional connectivity 
with all structures and connections sharing virtually the same state. Notably, in binge-drinking animals hardly any changes occurred, leaving the brain at functional plateau. Alcohol reexposure resulted in a major reduction in a number of links between these structures and fragmentation of functional correlations. The strongest connections among selected brain structures were found for BST and VTA that shared the same module, but also strongly correlated in terms of c-Fos activation. Of note, projections from neurons of BST to VTA are crucial to behaviors related to reward and motivation (Jalabert et al, 2009). Interestingly, another strong functional correlation was observed between GPe and BLA, and in particular BLAp, but not BLAa or BLAv, suggesting a dissociable role for posterior BLA in alcohol reexposure. Although much less is known of the function of the BLAp relative to BLAa, its role in cue-triggered alcohol-seeking behavior has been suggested (Millan et al, 2015). In humans, alcohol can change GPe activity by decreasing neuronal firing rates, suggesting that the GPe may have a central role in explaining impulsive behaviors during binge drinking (Fede et al, 2020). BLAp and GPe do not share any direct projection pathway, but they are indirectly connected via striatal neurons (Yager et al, 2015). Another strong connection was formed between HIP, SNc and CeA. The HIP is critical for the acquisition of new factual information and the formation of new memories about personally emotionally relevant experienced events (Eichenbaum, 2004), whereas CEA is related to memory consolidation for emotionally arousing events (Cahill et al, 1995; McGaugh et al, 1996). Furthermore, SNc dopamine neurons are involved in learning to predict which behavior will lead to a reward (Keiflin \& Janak, 2015). Surprisingly in our study ACB was not found to be functionally connected with any of other analyzed structures.

The present study employed a novel behavioral paradigm that was based on the IntelliCage system to investigate binge-like drinking in mice. We used a high-throughput method that was based on brain clearing followed by light-sheet microscopy imaging to assess 
the whole-brain response to alcohol. Specific brain structures were shown to specifically respond to alcohol craving. Alterations of overall functional brain organization, including an increase in modularity and brain network disruption, in the context of alcohol craving were also observed. 


\section{Materials and Methods}

Additional details of all procedures described below are available in the Supplementary Methods and Materials.

\section{Behavioral training}

The experiments were performed using female C57B16 mice that were divided into the four groups: control (CTRL group; $n=5$ ), binge-like drinking (BD group; $n=5$ ), cue reexposure (CR group; $n=5$ ) and alcohol reexposure (AR group; $n=5$ ). We used an animal model of binge-like alcohol drinking (Thiele \& Navarro, 2014) that was adapted to the IntelliCage system. The procedures were performed according to the Polish Animal Protection Act, Directive 2010/63/EU, and approved by the Local Ethics Committee (permission no. 128/2016).

Optical tissue clearing and whole-brain c-Fos imaging

Optical tissue clearing was performed using iDisco+ method (Renier et al., 2016; Renier et al., 2014). Brains were imaged using a light-sheet microscope (Pawlowska et al., 2019; Stefaniuk et al, 2016).

\section{Computational analysis}

We developed a workflow that included image registration techniques and cell detection algorithms. We took advantage of multichannel acquisitions that provided both functional and anatomical information. For each c-Fos-positive nucleus, a local maximum intensity was determined. The sum of the signal for each structure was next overlaid on its volume (in $\mathrm{mm}^{3}$ ) to create a signal density map.

\section{Statistical analysis}

The results are expressed as mean \pm SEM. Analyses were conducted using Prism 8.4.2 software (GraphPad, La Jolla, CA, USA). Differences between experimental groups were 
considered significant if the type 1 error was less than 5\%. For comparisons of the c-Fos signal density between structures, we first performed a mixed-effect linear model following by the analysis of variance (ANOVA). The between-group differences were assessed by the two-way paired $t$-tests. Obtained $p$ values were corrected for multiple comparison to $q$ values (Ryabinin et al., 1997). The tests were performed in Python 3.7 using the Pingouin 0.3.3 statistical package and custom-written scripts (source code and data can be found at https://github.com/pawlowska/pairwise tests pingouin). The network analysis was conducted using igraph package for R statistical environment; figures were prepared in Cytoscape 3.8.2 software.

\section{Acknowledgments}

This work was supported by a TEAM grant (POIR.04.04.00-00-1ACA/16-00). MS was supported by a National Science Centre grant (2019/35/B/NZ4/04077). MP was supported by a National Science Centre grant (UMO-2015/17/B/NZ4/02631). PM and DW were supported by an ERA-NET NEURON grant from the National Centre for Research and Development (ERA-NET-NEURON/17/2017). The authors thank Prof. Kasia Radwanska and Dr. Anna Beroun for constructive criticism of the manuscript and Michael Arends for proofreading,

\section{Author contributions}

MS and MP designed experiments and wrote the manuscript, MS and KN performed behavioral experiments, MS performed imaging, MP constructed a light-sheet set-up and performed image and computational analysis, LB and DL optimized the protocol and performed the clearing, $\mathrm{PM}, \mathrm{SB}, \mathrm{NJ}, \mathrm{ZZ}$ and $\mathrm{MB}$ performed computational analysis, $\mathrm{DW}$ and LK supervised the work

\section{Conflict of interest}


The authors declare that they have no conflict of interest.

\section{Data Availability Section}

Datasets and codes generated and used in the reported study are listed in Supplemental Information and attached as a Supplementary File. 


\section{References}

(2004) NIAAA Council Approves Definition of Binge Drinking. NIAAA Newsletter 3: 4 (2017) Allen Mouse Common Coordinate Framework and Reference Atlas. Alleninstituteorg Arnemann KL, Chen AJW, Novakovic-Agopian T, Gratton C, Nomura EM, D'Esposito M (2015) Functional brain network modularity predicts response to cognitive training after brain injury. Neurology 84: 1568-1574

Augier E, Barbier E, Dulman RS, Licheri V, Augier G, Domi E, Barchiesi R, Farris S, Natt D, Mayfield RD et al (2018) A molecular mechanism for choosing alcohol over an alternative reward. Science 360: 1321-1326

Belin D, Belin-Rauscent A, Murray JE, Everitt BJ (2013) Addiction: failure of control over maladaptive incentive habits. Curr Opin Neurobiol 23: 564-572

Beroun A, Nalberczak-Skora M, Harda Z, Piechota M, Ziolkowska M, Caly A, Pagano R, Radwanska K (2018) Generation of silent synapses in dentate gyrus correlates with development of alcohol addiction. Neuropsychopharmacol 43: 1989-1999

Bullmore E, Sporns O (2009) Complex brain networks: graph theoretical analysis of structural and functional systems. Nat Rev Neurosci 10: 186-198

Cahill L, Babinsky R, Markowitsch HJ, McGaugh JL (1995) The amygdala and emotional memory. Nature 377: 295-296

Chung K, Wallace J, Kim SY, Kalyanasundaram S, Andalman AS, Davidson TJ, Mirzabekov JJ, Zalocusky KA, Mattis J, Denisin AK et al (2013) Structural and molecular interrogation of intact biological systems. Nature 497: 332-337

Cox BR, Olney JJ, Lowery-Gionta EG, Sprow GM, Rinker JA, Navarro M, Kash TL, Thiele TE (2013) Repeated cycles of binge-like ethanol (EtOH)-drinking in male C57BL/6J mice augments subsequent voluntary EtOH intake but not other dependence-like phenotypes. Alcohol Clin Exp Res 37: 1688-1695

Dawson DA, Grant BF, Li TK (2005) Quantifying the risks associated with exceeding recommended drinking limits. Alcohol Clin Exp Res 29: 902-908

Eichenbaum H (2004) Hippocampus: cognitive processes and neural representations that underlie declarative memory. Neuron 44: 109-120

Fede SJ, Abrahao KP, Cortes CR, Grodin EN, Schwandt ML, George DT, Diazgranados N, Ramchandani VA, Lovinger DM, Momenan R (2020) Alcohol effects on globus pallidus connectivity: Role of impulsivity and binge drinking. Plos One 15: e0224906

Fuchs RA, Higginbotham JA, Hansen EJ (2019) Animal Models of Addiction. Neural Mechanisms of Addiction: 3-22

George O, Hope BT (2017) Cortical and amygdalar neuronal ensembles in alcohol seeking, drinking and withdrawal. Neuropharmacology 122: 107-114

George O, Koob GF (2010) Individual differences in prefrontal cortex function and the transition from drug use to drug dependence. Neurosci Biobehav Rev 35: 232-247

Giardino WJ, Ryabinin AE (2013) CRF1 receptor signaling regulates food and fluid intake in the drinking-in-the-dark model of binge alcohol consumption. Alcoholism Clinical and Experimental Research 37: 1161-1170

Giuliano C, Pena-Oliver Y, Goodlett CR, Cardinal RN, Robbins TW, Bullmore ET, Belin D, Everitt BJ (2018) Evidence for a long-lasting compulsive alcohol seeking phenotype in rats. Neuropsychopharmacol 43: 728-738

Goldstein RZ, Volkow ND (2002) Drug addiction and its underlying neurobiological basis: neuroimaging evidence for the involvement of the frontal cortex. Am J Psychiatry 159: 1642-1652

Guimera R, Nunes Amaral LA (2005) Functional cartography of complex metabolic networks. Nature 433: 895-900

Hamlin AS, Clemens KJ, Choi EA, McNally GP (2009) Paraventricular thalamus mediates contextinduced reinstatement (renewal) of extinguished reward seeking. Eur J Neurosci 29: 802-812

Hamlin AS, Newby J, McNally GP (2007) The neural correlates and role of D1 dopamine receptors in renewal of extinguished alcohol-seeking. Neuroscience 146: 525-536 
Jalabert M, Aston-Jones G, Herzog E, Manzoni O, Georges F (2009) Role of the bed nucleus of the stria terminalis in the control of ventral tegmental area dopamine neurons. Prog Neuropsychopharmacol Biol Psychiatry 33: 1336-1346

Keiflin R, Janak PH (2015) Dopamine Prediction Errors in Reward Learning and Addiction: From Theory to Neural Circuitry. Neuron 88: 247-263

Keyes PC, Adams EL, Chen Z, Bi L, Nachtrab G, Wang VJ, Tessier-Lavigne M, Zhu Y, Chen X (2020) Orchestrating Opiate-Associated Memories in Thalamic Circuits. Neuron

Kimbrough A, Lurie DJ, Collazo A, Kreifeldt M, Sidhu H, Macedo GC, D'Esposito M, Contet C, George O (2020) Brain-wide functional architecture remodeling by alcohol dependence and abstinence. Proc Natl Acad Sci U S A 117: 2149-2159

Kiryk A, Janusz A, Zglinicki B, Turkes E, Knapska E, Konopka W, Lipp HP, Kaczmarek L (2020) IntelliCage as a tool for measuring mouse behavior-20 years perspective. Behavioural Brain Research 388

Knapska E, Radwanska K, Werka T, Kaczmarek L (2007) Functional internal complexity of amygdala: focus on gene activity mapping after behavioral training and drugs of abuse. Physiol Rev 87: 1113-1173

Knutson B, Westdorp A, Kaiser E, Hommer D (2000) FMRI visualization of brain activity during a monetary incentive delay task. Neuroimage 12: 20-27

Koob GF, Volkow ND (2016) Neurobiology of addiction: a neurocircuitry analysis. Lancet Psychiat 3: 760-773

Koskela M, Piepponen TP, Andressoo JO, Voikar V, Airavaara M (2018) Towards developing a model to study alcohol drinking and craving in female mice housed in automated cages. Behav Brain Res 352: 116-124

Liu R, Chen H, Qin R, Gu Y, Chen X, Zou J, Jiang Y, Li W, Bai F, Zhang B et al (2019) The Altered Reconfiguration Pattern of Brain Modular Architecture Regulates Cognitive Function in Cerebral Small Vessel Disease. Front Neurol 10: 324

Luscher C, Robbins TW, Everitt BJ (2020) The transition to compulsion in addiction. Nature Reviews Neuroscience 21: 247-263

Marinelli PW, Funk D, Juzytsch W, Li Z, Le AD (2007) Effects of opioid receptor blockade on the renewal of alcohol seeking induced by context: relationship to c-fos mRNA expression. Eur $J$ Neurosci 26: 2815-2823

Martin C (2014) Contributions and complexities from the use of in vivo animal models to improve understanding of human neuroimaging signals. Front Neurosci 8: 211

McGaugh JL, Cahill L, Roozendaal B (1996) Involvement of the amygdala in memory storage: interaction with other brain systems. Proc Natl Acad Sci U S A 93: 13508-13514

Mesulam MM (1990) Large-scale neurocognitive networks and distributed processing for attention, language, and memory. Ann Neurol 28: 597-613

Meunier D, Lambiotte R, Bullmore ET (2010) Modular and hierarchically modular organization of brain networks. Front Neurosci-Switz 4

Meunier D, Lambiotte R, Fornito A, Ersche KD, Bullmore ET (2009) Hierarchical modularity in human brain functional networks. Front Neuroinform 3: 37

Millan EZ, Furlong TM, McNally GP (2010) Accumbens Shell-Hypothalamus Interactions Mediate Extinction of Alcohol Seeking. J Neurosci 30: 4626-4635

Millan EZ, Reese RM, Grossman CD, Chaudhri N, Janak PH (2015) Nucleus Accumbens and Posterior Amygdala Mediate Cue-Triggered Alcohol Seeking and Suppress Behavior During the Omission of Alcohol-Predictive Cues. Neuropsychopharmacol 40: 2555-2565

Namba MD, Tomek SE, Olive MF, Beckmann JS, Gipson CD (2018) The Winding Road to Relapse: Forging a New Understanding of Cue-Induced Reinstatement Models and Their Associated Neural Mechanisms. Front Behav Neurosci 12: 17

Nestler EJ (2001) Molecular basis of long-term plasticity underlying addiction (vol 2, pg 119, 2001). Nature Reviews Neuroscience 2: 215-215

Newman ME (2006) Modularity and community structure in networks. P Natl Acad Sci USA 103: 8577-8582

Pawlowska M, Stefaniuk M, Legutko D, Kaczmarek L (2019) Light-Sheet Microscopy for WholeBrain Imaging. Prog Opt Sci Photon 5: 69-81 
Radwanska K, Kaczmarek L (2012) Characterization of an alcohol addiction-prone phenotype in mice. Addict Biol 17: 601-612

Radwanska K, Wrobel E, Korkosz A, Rogowski A, Kostowski W, Bienkowski P, Kaczmarek L (2008) Alcohol relapse induced by discrete cues activates components of AP-1 transcription factor and ERK pathway in the rat basolateral and central amygdala. Neuropsychopharmacol 33: 1835-1846

Renier N, Adams EL, Kirst C, Wu ZH, Azevedo R, Kohl J, Autry AE, Kadiri L, Venkataraju KU, Zhou Y et al (2016) Mapping of brain activity by automated volume analysis of immediate early genes. Cell 165: 1789-1802

Renier N, Wu Z, Simon DJ, Yang J, Ariel P, Tessier-Lavigne M (2014) iDISCO: a simple, rapid method to immunolabel large tissue samples for volume imaging. Cell 159: 896-910

Rhodes JS, Best K, Belknap JK, Finn DA, Crabbe JC (2005) Evaluation of a simple model of ethanol drinking to intoxication in C57BL/6J mice. Physiol Behav 84: 53-63

Rhodes JS, Ford MM, Yu CH, Brown LL, Finn DA, Garland T, Crabbe JC (2007) Mouse inbred strain differences in ethanol drinking to intoxication. Genes Brain Behav 6: 1-18

Rubinov M, Sporns O (2010) Complex network measures of brain connectivity: uses and interpretations. Neuroimage 52: 1059-1069

Ryabinin AE, Criado JR, Henriksen SJ, Bloom FE, Wilson MC (1997) Differential sensitivity of c-Fos expression in hippocampus and other brain regions to moderate and low doses of alcohol. $\mathrm{Mol}$ Psychiatry 2: 32-43

Sanchis-Segura C, Spanagel R (2006) Behavioural assessment of drug reinforcement and addictive features in rodents: an overview. Addict Biol 11: 2-38

Schneeberger M, Parolari L, Das Banerjee T, Bhave V, Wang P, Patel B, Topilko T, Wu Z, Choi CHJ, Yu X et al (2019) Regulation of Energy Expenditure by Brainstem GABA Neurons. Cell 178: 672-685 e612

Skora MN, Pattij T, Beroun A, Kogias G, Mielenz D, de Vries T, Radwanska K, Muller CP (2020) Personality driven alcohol and drug abuse: New mechanisms revealed. Neurosci Biobehav Rev 116: 64-73

Smith RJ, Anderson RI, Haun HL, Mulholland PJ, Griffin WC, Lopez MF, Becker HC (2019) Dynamic c-Fos changes in mouse brain during acute and protracted withdrawal from chronic intermittent ethanol exposure and relapse drinking. Addiction Biology

Smutek M, Turbasa M, Sikora M, Piechota M, Zajdel J, Przewlocki R, Parkitna JR (2014) A model of alcohol drinking under an intermittent access schedule using group-housed mice. Plos One 9

Spanagel R (2017) Animal models of addiction. Dialogues Clin Neurosci 19: 247-258

Sporns O (2010) Networks of the Brain. MIT Press

Sporns O, Betzel RF (2016) Modular Brain Networks. Annu Rev Psychol 67: 613-640

Stefaniuk M, Beroun A, Lebitko T, Markina O, Leski S, Meyza K, Grzywacz A, Samochowiec J, Samochowiec A, Radwanska K et al (2017) Matrix Metalloproteinase-9 and synaptic plasticity in the central amygdala in control of alcohol-seeking behavior. Biol Psychiatry 81: 907-917

Stefaniuk M, Gualda EJ, Pawlowska M, Legutko D, Matryba P, Koza P, Konopka W, Owczarek D, Wawrzyniak M, Loza-Alvarez P et al (2016) Light-sheet microscopy imaging of a whole cleared rat brain with Thy1-GFP transgene. Sci Rep-Uk 6

Stevens AA, Tappon SC, Garg A, Fair DA (2012) Functional Brain Network Modularity Captures Inter- and Intra-Individual Variation in Working Memory Capacity. Plos One 7

Sun X, Bernstein MJ, Meng M, Rao S, Sorensen AT, Yao L, Zhang X, Anikeeva PO, Lin Y (2020) Functionally Distinct Neuronal Ensembles within the Memory Engram. Cell 181: 410-423 e417

Susaki EA, Tainaka K, Perrin D, Yukinaga H, Kuno A, Ueda HR (2015) Advanced CUBIC protocols for whole-brain and whole-body clearing and imaging. Nat Protoc 10: 1709-1727

Thiele TE, Navarro M (2014) "Drinking in the dark" (DID) procedures: a model of binge-like ethanol drinking in non-dependent mice. Alcohol 48: 235-241

Wilcox MV, Carlson VCC, Sherazee N, Sprow GM, Bock R, Thiele TE, Lovinger DM, Alvarez VA (2014) Repeated binge-like ethanol drinking alters ethanol drinking patterns and depresses striatal GABAergic transmission Neuropsychopharmacol 39: 2039-2040

Yager LM, Garcia AF, Wunsch AM, Ferguson SM (2015) The ins and outs of the striatum: role in drug addiction. Neuroscience 301: 529-541 
Yap EL, Pettit NL, Davis CP, Nagy MA, Harmin DA, Golden E, Dagliyan O, Lin C, Rudolph S, Sharma N et al (2020) Bidirectional perisomatic inhibitory plasticity of a Fos neuronal network. Nature

Zahr NM, Pfefferbaum A (2017) Alcohol's Effects on the Brain: Neuroimaging Results in Humans and Animal Models. Alcohol Res 38: 183-206

Zhang R, Volkow ND (2019) Brain default-mode network dysfunction in addiction. Neuroimage 200: 313-331 


\section{Figures}

A Experimental design $\quad$ BD - binge-drinkers, CR - cue reexposure, AR - alcohol reexposure

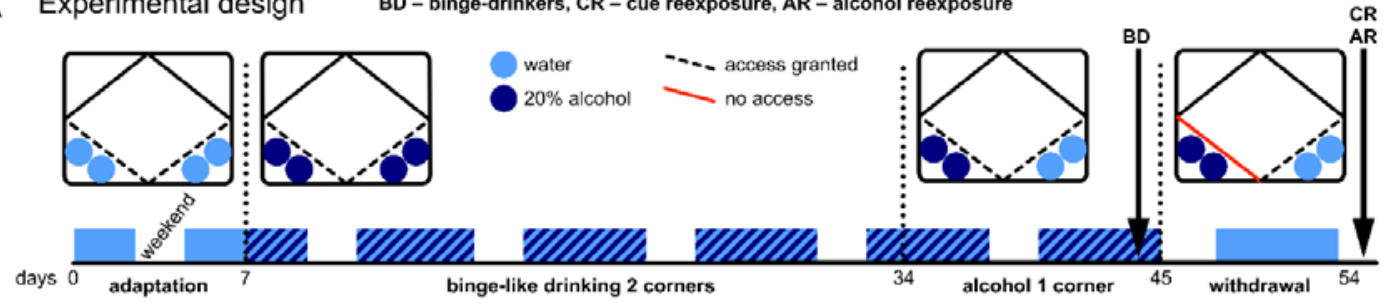

B IntelliCage corner
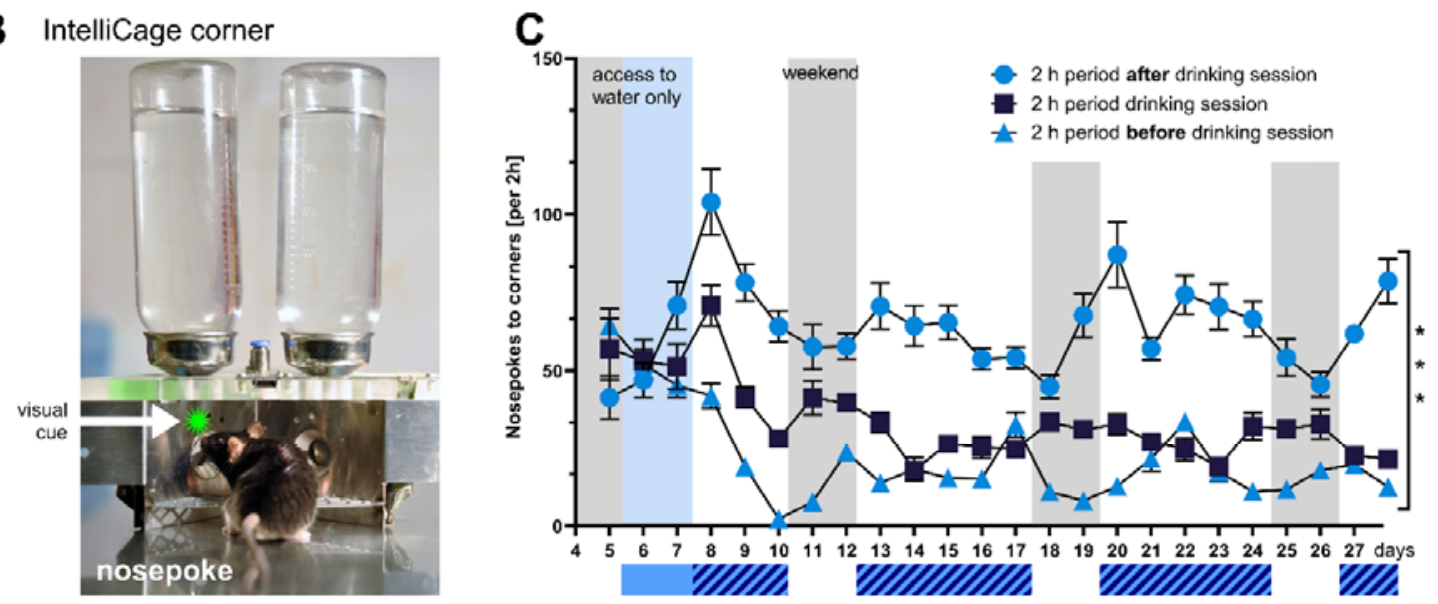

D



E

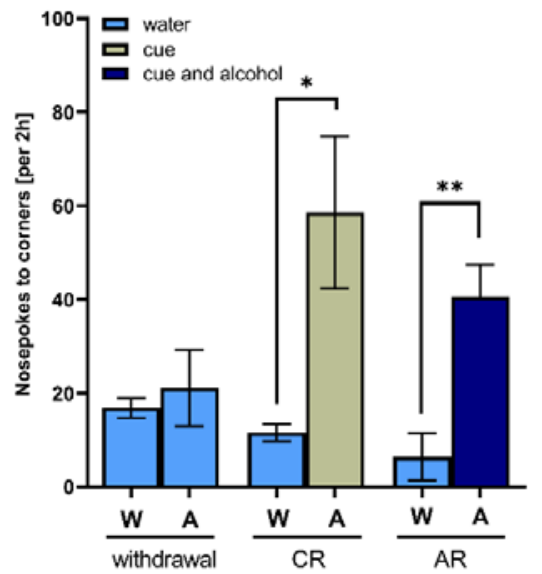

Figure 1. Binge-like alcohol drinking in the IntelliCage. (A) Experimental design and cage configuration. Blue circle: access to water. Dark blue circle: access to alcohol. Dashed line: free access. Solid red line: no access. (B) IntelliCage corner. The mice had access to two corners that were equipped with a door that controlled access to the bottles. Whenever access to alcohol was granted, a visual cue (a green LED over the door) turned on. (C) Nosepokes in the corners at 2-h intervals. The mice actively sought alcohol when no access to alcohol was 
granted (nosepokes in the corners, two-way ANOVA, $F_{2,1005}=511, * * * p<0.0001$ ). For each day, three time intervals are presented: blue triangle (2-h period before alcohol access), dark blue square ( $2 \mathrm{~h}$ of alcohol access in DID session), blue circle (2-h period after $2 \mathrm{~h}$ of alcohol access). The data are expressed as mean \pm SEM. (D) Increase in the number of nosepokes in corners when access to alcohol was ceased (two-way ANOVA, $F_{2,945}=367.8$, $* * * p<0.0001$ ). (E) Behavioral phenotyping upon reexposure. The mice made more nosepokes in the corner with alcohol following reexposure compared with the water corner in CR group $p=0.0206, t_{8}=2,878$ and AR group $p=0.0015, t_{8}=4,746 . \mathrm{W}$, water corner; $\mathrm{A}$, alcohol corner (during withdrawal, A indicates the corner with the previous alcohol access); $\mathrm{CR}$, cue reexposure; AR, alcohol reexposure. The data are expressed as mean $\pm \mathrm{SEM} .{ }^{*} p<$ $0.05, * * p<0.01(t$-test $)$ 

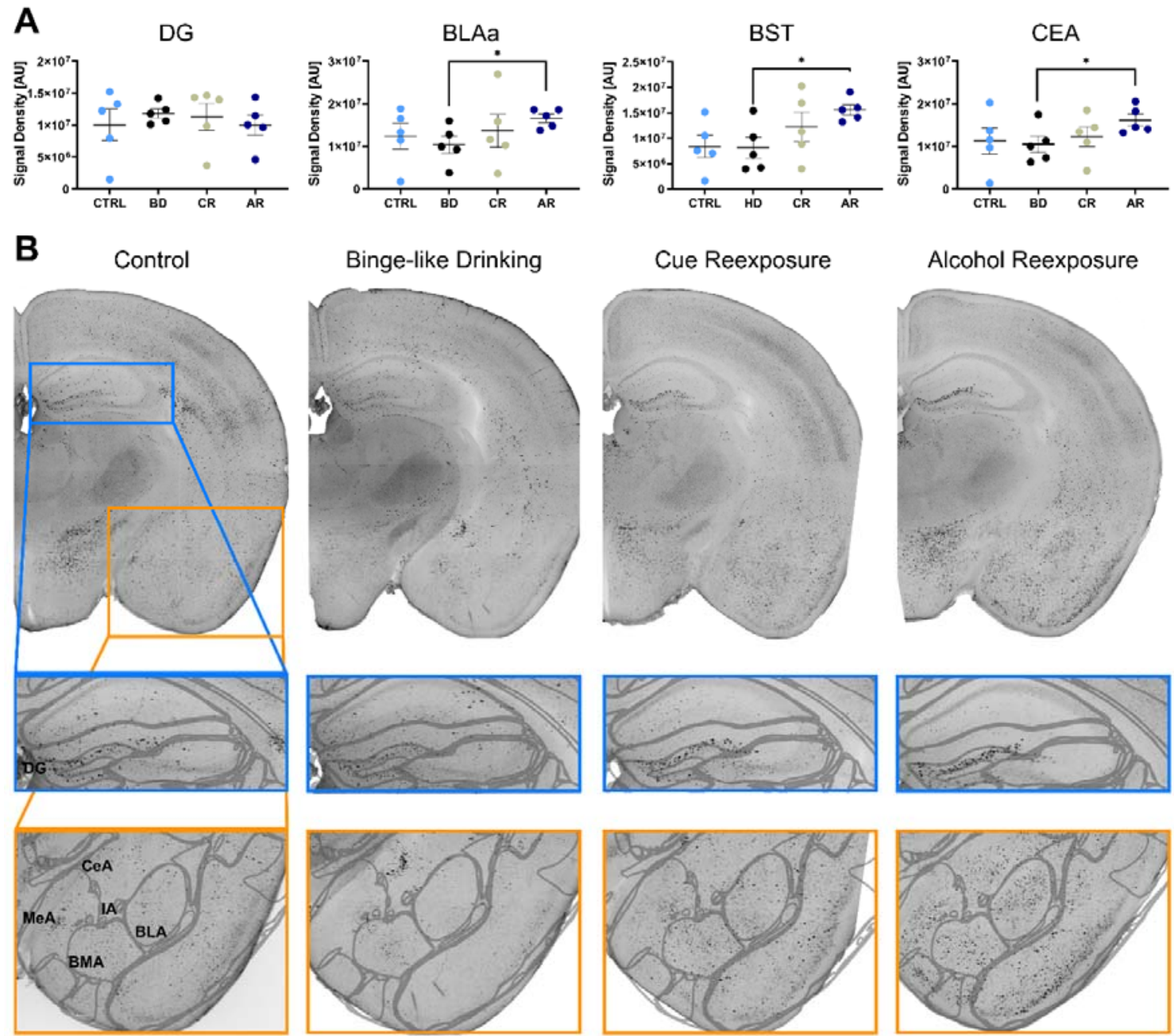

Alcohol Reexposure
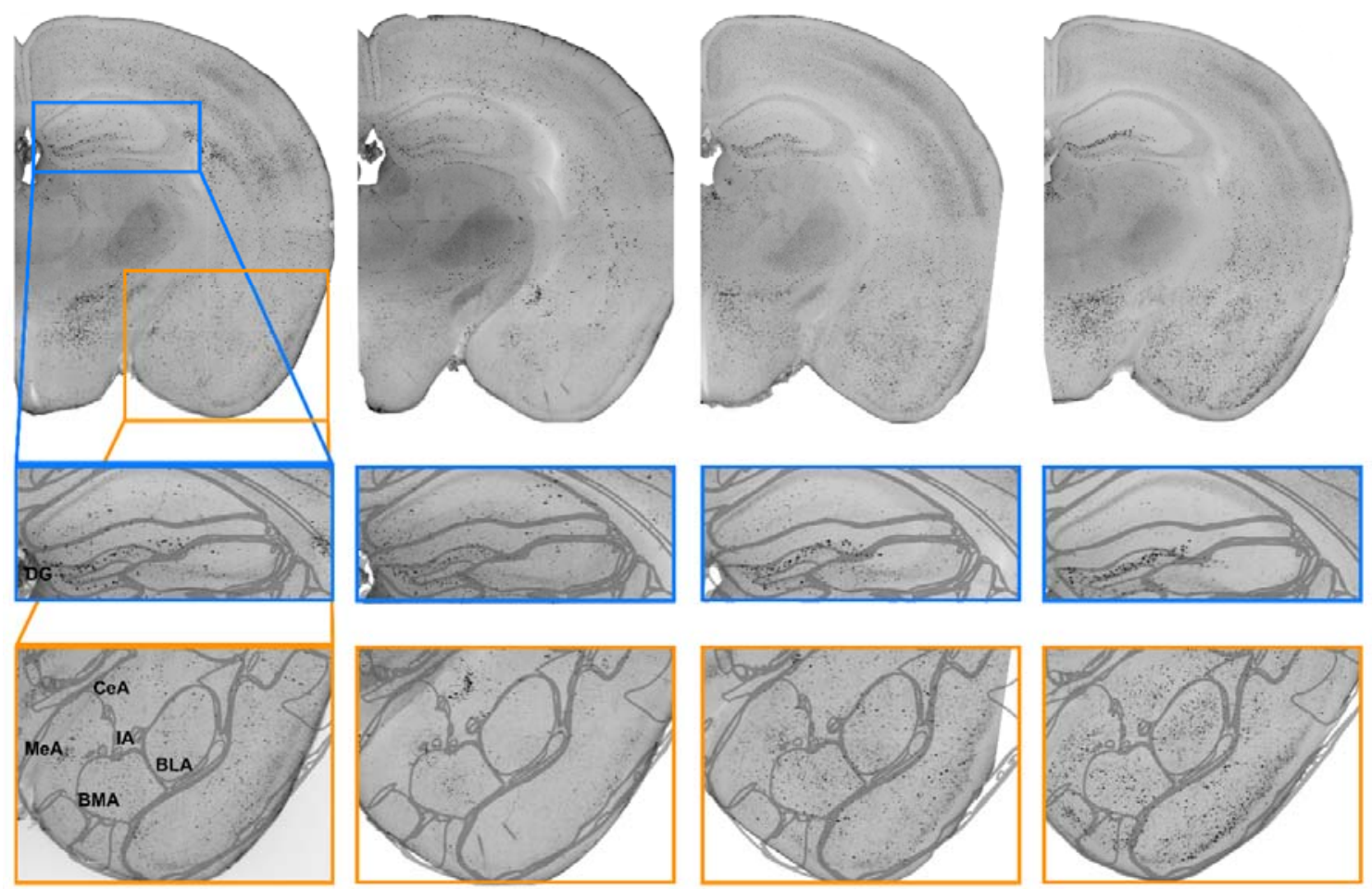

Figure 2. Overview of profile of c-Fos expression in selected brain structures. (A) The signal density for each structure was obtained by dividing the sum of the signals of individual c-Fospositive cells in a given structure by its volume (in $\mathrm{mm}^{3}$ ). $* p<0.05$, **p $<0.01$, $* * * p<0.001$ ( $t$-test). No difference in the c-Fos signal density was found in the dentate gyrus between experimental groups and increase in signal density in selected amygdala nuclei and BST was observed. (B) Coronal sections of maximum intensity projections that covered $50 \mu \mathrm{m}$, reconstructed from whole-brain light-sheet microscopy images. Single c-Fos-positive nuclei in specific brain areas and differences between experimental groups are shown. BLA, basolateral amygdala; BST, bed nucleus of stria terminalis; CeA, central nucleus of the amygdala; DG, dentate gyrus; MeA, medial amygdala; Control, control naive group with 

available under aCC-BY-NC-ND 4.0 International license.

access to water only; Binge-like Drinking group with no history of alcohol withdrawal; Cue

Reexposure, alcohol-related cue reexposure group; Alcohol Reexposure, alcohol reexposure group. 




Figure 3. Overview of the c-Fos signal density profile in the whole brain, showing a comparison between the binge-like drinking group and alcohol reexposure group. The signal density for each structure was obtained by dividing the sum of signals of individual c-Fospositive cells in a given structure by its volume (in $\mathrm{mm}^{3}$ ). The structures are grouped in 
anatomical categories according to the Allen Brain Atlas. Within-category structures are shown according to decreasing signal density. The data were analyzed using one-way paired $t$-test, and resulting $p$ values are in brackets next to the structure acronym. 
bioRxiv preprint doi: https://doi.org/10.1101/2021.02.17.431586; this version posted March 1, 2021. The copyright holder for this preprint (which was not certified by peer review) is the author/funder, who has granted bioRxiv a license to display the preprint in perpetuity. It is made available under aCC-BY-NC-ND 4.0 International license.

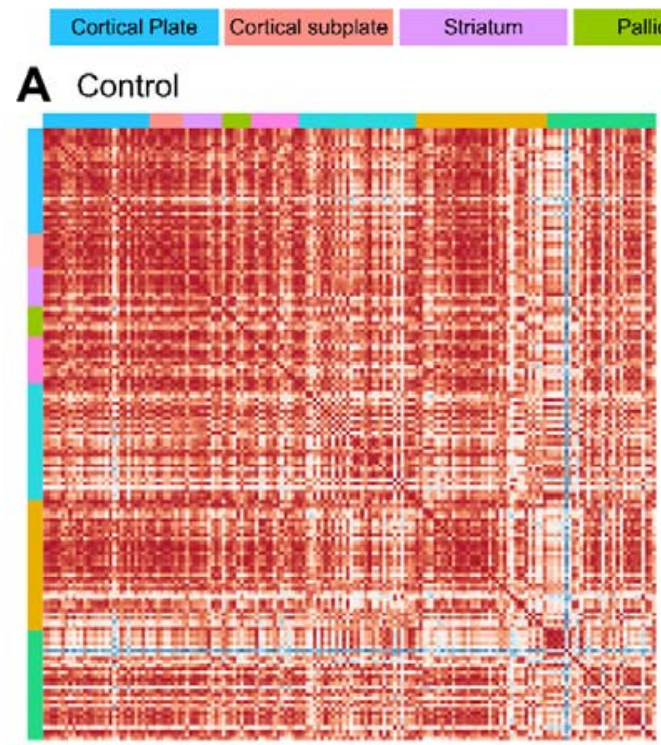

\section{Cue Reexposure}

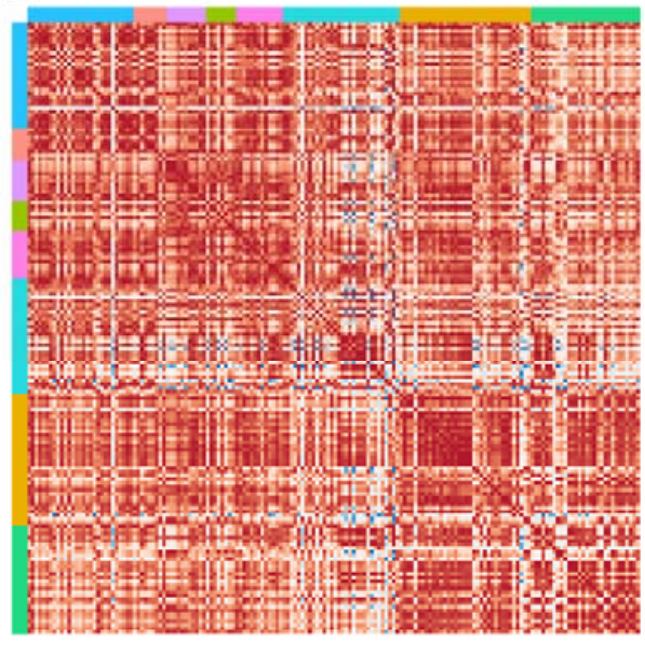

\begin{tabular}{|l|l|l|l|}
\hline Thalamus & Hypothalamus & Midbrain & Hindbrain \\
\hline
\end{tabular}

B Binge-like Drinking

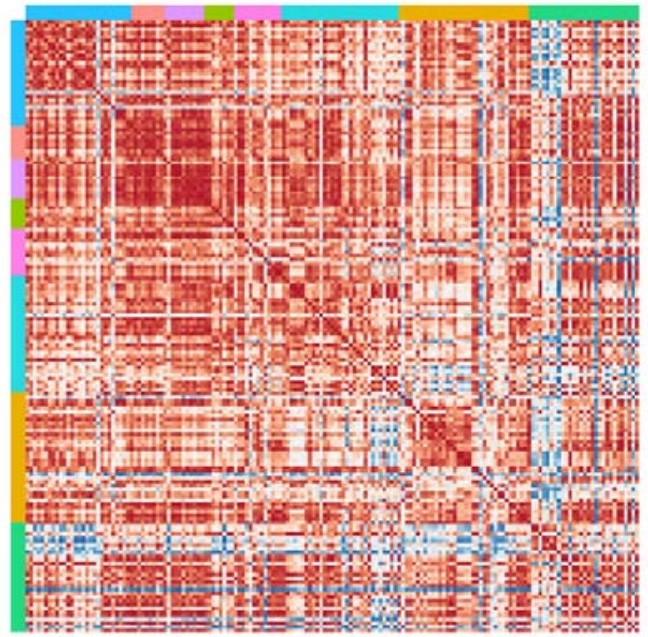

\section{Alcohol Reexposure}

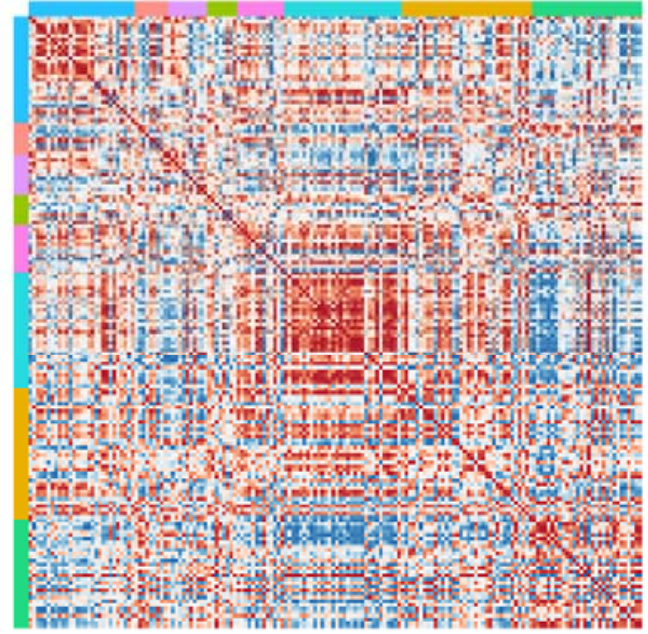

E
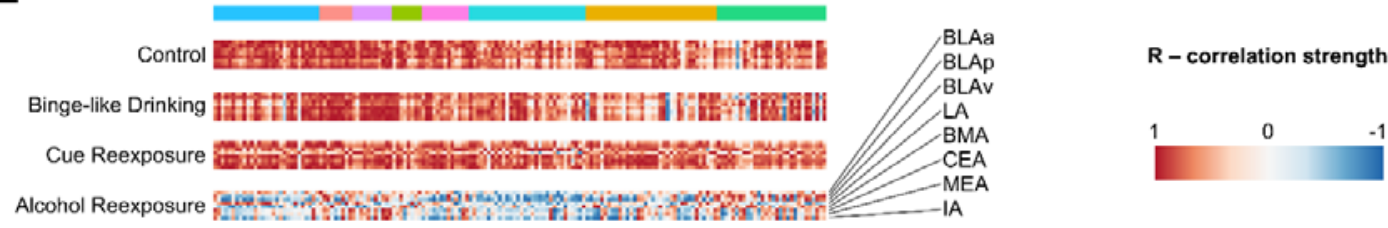

Figure 4. Brain region correlation heatmaps organized according to the Allen Brain Atlas. (A)

Correlation map for the naive control group that had access to water only and no previous exposure to alcohol. (B) Correlation map for the binge drinking group whose brains were snap frozen after the last drinking session. (C) Correlation map for the cue-reexposed group following alcohol withdrawal. (D) Correlation map for the alcohol reexposure group 
following alcohol withdrawal. Color-coded anatomical annotations are depicted above the heatmaps. $\mathrm{R}$ indicates the correlation strength between single structures, in which warmer colors indicate more similar signal densities between two structures in all mice $(n=5)$ in a given group. Cooler colors indicate a lower correlation. (E) Comparison of correlations of amygdala structures $v s$. all other regions. 
bioRxiv preprint doi: https://doi.org/10 1101/2021.02 17.431586; this version posted March 1, 2021. The copyright holder for this preprint (which was not certified by peer review) is the author/funder, who has granted bioRxiv a license to display the preprint in perpetuity. It is made available under aCC-BY-NC-ND 4.0 International license.

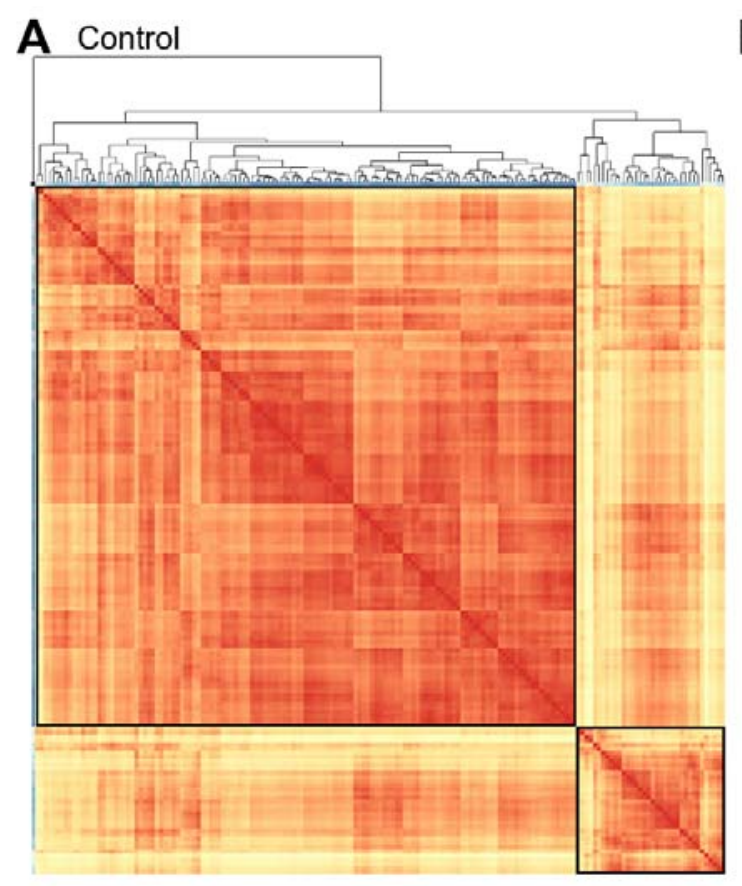

\section{B Binge-like Drinking}

\section{Cue Reexposure}
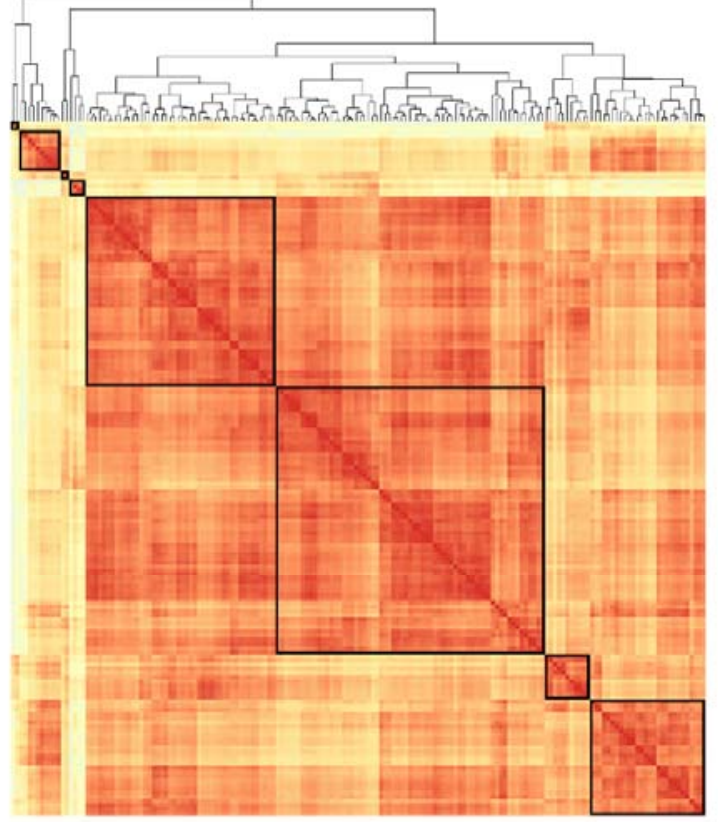

\section{Alcohol Reexposure}

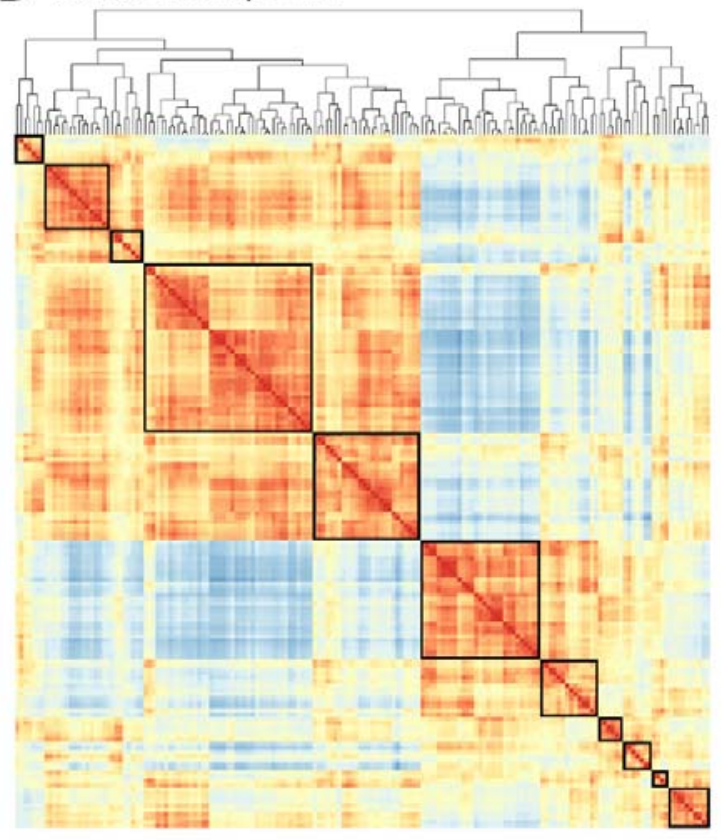

\section{$\mathbf{E}$}

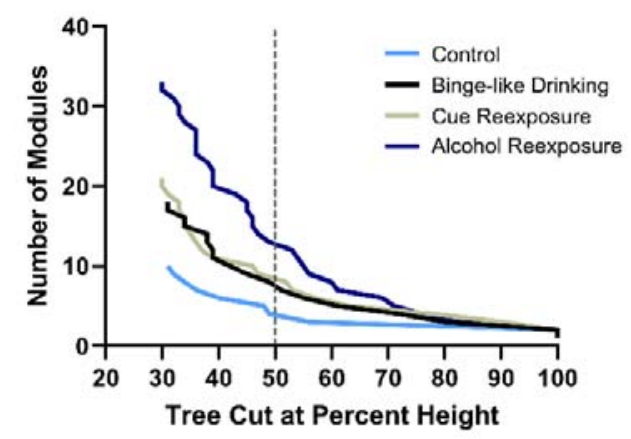

Euclidean distance 
Figure 5. Intra-network hierarchical organization of sub-networks, showing the relative distance between brain regions in the (A) control group, (B) binge-like drinking group, (C) cue reexposure group, and (D) alcohol reexposure group. The Euclidean distance was calculated to create the dendrograms. The lines in the dendrograms are drawn in proportion to the distance measure of the nodes. The sub-networks are in ascending order according to their distances in the sub-dendrogram. Warmer colors indicate a shorter Euclidean distance, indicating that two structures have highly similar c-Fos signal density correlation patterns with all other structures. Black squares indicate modules that were created by cutting the dendrograms at half the maximal tree height. (E) Number of modules per group based on cutting the dendrograms at different percentages of tree heights. The dashed line indicates cutting at $50 \%$ to create modules in A-D. 

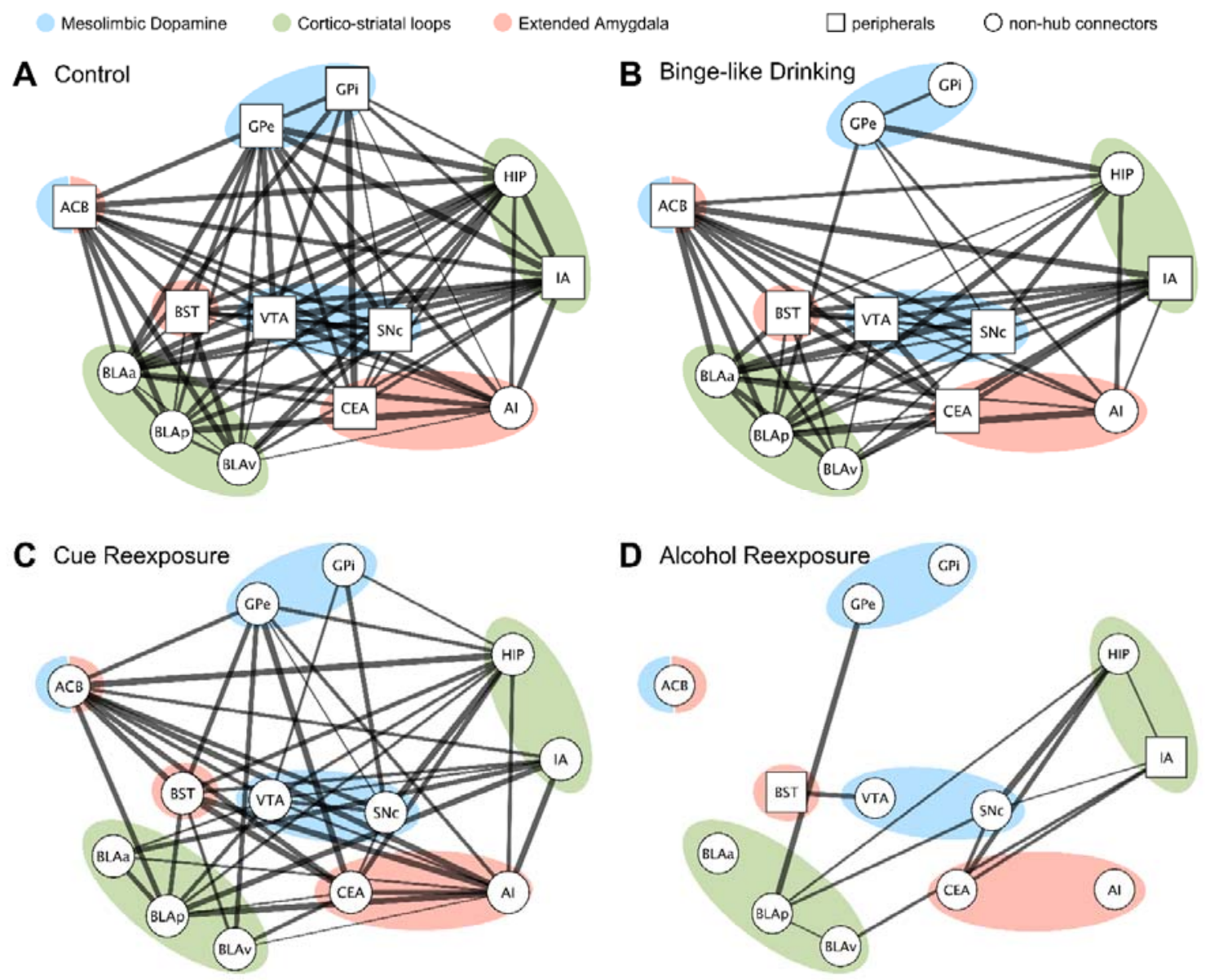

Figure 6. Functional connectivity of brain regions relevant to addiction. Each node (square or circle) represents a structure. The shape of the node represents the importance of node within module and between modules (see Supplementary Figure ) with non-hub connectors (circles) being important connecting different modules and peripherals having equal role within and outside module. The thickness of the lines (edges) represents the strength of the correlation, with thicker line representing higher correlation. Gross anatomy is represented by colored bubble areas. Note loss of edges in binge-like drinking animals (B) and further in cue (C) and alcohol reexposed animals (D) when compared to control animals (A) indicating alterations of functional interactions from disruption to strengthening.

Structures selected from (Koob \& Volkow, 2016). For abbreviations see Supplementary Dataset 1. 